\title{
Effets des dépenses publiques d'éducation, la fiscalité et la corruption sur la croissance économique en Afrique de l'ouest : une analyse empirique sur données de panel
}

\author{
Thierno Ndao Guèye \\ UFR Sciences Économiques et de Gestion \\ Université Assane Seck de Ziguinchor, Sénégal
}

Doi:10.19044/esj.2021.v17n38p160

Submitted: 20 September 2021

Accepted: 13 November 2021

Published: 30 November 2021
Copyright 2021 Author(s)

Under Creative Commons BY-NC-ND

4.0 OPEN ACCESS

Cite As:

Guèye T.N. (2021). Effets des dépenses publiques d'éducation, la fiscalité et la corruption sur la croissance économique en Afrique de l'ouest : une analyse empirique sur données de panel. European Scientific Journal, ESJ, 17 (38), 160.

https://doi.org/10.19044/esj.2021.v17n38p160

\section{Résumé}

Dans cette étude, nous examinons empiriquement les effets des dépenses publiques d'éducation, la fiscalité et la corruption sur la croissance économique par habitant à long terme à partir de modèles économétriques des données de panel de 10 pays d'Afrique de l'ouest sur la période 2004-2020. D'abord, nos résultats montrent qu'il existe une relation positive et significative entre les dépenses publiques d'éducation retardées de 5 ans et la croissance économique par habitant. En effet, une augmentation de $1 \%$ des dépenses publiques d'éducation entraînerait une hausse moyenne de $0,42 \%$ du taux de croissance économique. Tous les gouvernements de l'Afrique occidentale veulent atteindre les objectifs de l'éducation pour tous. Cette extension ne pourrait pas se réaliser sans une augmentation des dépenses publiques. Parallèlement, suivant l'approche de l'équivalence ricardienne, les sources de financement de l'éducation, telles que les impôts et les dettes publiques, agissent négativement sur la croissance économique. Ensuite, la corruption, en affectant significativement la croissance économique, entraîne des pertes importantes de recettes fiscales. Ce qui gangrène l'efficacité des efforts de financement des politiques publiques d'éducation. Enfin, dans le cadre scolaire global, seul le taux de scolarisation aux études supérieures exerce un effet réellement significatif sur la croissance à long terme. Ce résultat est d'une grande portée, car la forte tertiarisation des économies de ces 
pays est accompagnée d'un secteur informel occupant une place très importante et marginalement pris en compte dans le calcul du PIB.

Mots clés : Dépenses publiques d'éducation, fiscalité, corruption, croissance économique, Afrique de l'ouest, données de panel

\title{
Effects of Public Education Spending, Taxation, and Corruption on Economic Growth in West Africa: An Empirical Panel Data Analysis
}

\author{
Thierno Ndao Guèye \\ UFR Sciences Économiques et de Gestion \\ Université Assane Seck de Ziguinchor, Sénégal
}

\begin{abstract}
In this study, we empirically examine the effects of public education spending, taxation and corruption on long-term per capita economic growth using econometric models of panel data from 10 West African countries on the period 2004-2020. First, our results show that there is a positive and significant relationship between public education spending delayed by 5 years and per capita economic growth. Indeed, a $1 \%$ increase in public education spending would lead to an average $0.42 \%$ increase in the rate of economic growth. All governments in West Africa want to achieve education for all goals. This extension could not be achieved without an increase in public spending. At the same time, following the Ricardian equivalence approach, the sources of financing of education, such as taxes and public debt, act negatively on economic growth. Second, corruption, by significantly affecting economic growth, leads to significant losses in tax revenue. This undermines the effectiveness of efforts to fund public education policies. Finally, within the overall educational framework, only the rate of enrollment in higher education has a truly significant effect on long-term growth. This result is farreaching, because the strong tertiarization of the economies of these countries is accompanied by an informal sector occupying a very important place and marginally taken into account in the calculation of the GDP.
\end{abstract}

Keywords: Public expenditure on education, economic growth, taxation, corruption, West Africa, panel data 


\section{Introduction}

Les gouvernements du monde entier ont reconnu l'effet positif de l'éducation sur le développement économique. L'éducation permet de lutter contre la pauvreté, d'augmenter la productivité et le revenu individuel, trois facteurs essentiels dans tout processus de développement d'un pays. C'est pourquoi, la Déclaration d'Incheon adoptée en 2015 recommande que les pouvoirs publics consacrent $4 \%$ à $6 \%$ de leur PIB et/ou au moins $15 \%$ à $20 \%$ du montant total de leurs dépenses publiques à l'éducation, en mettant l'accent sur l'éducation de base (Note d'orientation 2018, Partenariat mondial pour l'éducation, GPE). Malheureusement, le contexte actuel est marqué par une crise réelle de financement de l'éducation et des difficultés pour les gouvernements à mobiliser des ressources publiques internes. En 2020, les dépenses publiques médianes des États pour l'éducation représentent 13,8\% des dépenses publiques (Rapport mondial de suivi de l'éducation 2020, UNESCO). Depuis 2005, nous assistons à une baisse régulière et significative de l'assistance des bailleurs bilatéraux à l'éducation de base en Afrique. Selon la Banque Mondiale (2020d), le total des engagements pour le financement du secteur de l'éducation en Afrique de l'ouest est estimé à 427 millions \$US en 2020 contre 164 millions \$US en 2021. Toutefois, la baisse graduelle des ressources pour financer les dépenses d'éducation est accompagnée d'une certaine disparité dans sa répartition. Dans les pays d'Amérique du Nord et d'Europe, les dépenses par élève dans le primaire sont équivalentes à celles de l'enseignement supérieur. En Afrique subsaharienne, non seulement les trois objectifs $^{1}$ quantifiables de l'Éducation Pour Tous (EPT) ne sont pas atteints, mais les dépenses par élève sont parfois dix fois plus élevées dans le supérieur que dans le primaire. En plus, dans ces pays, le secteur de l'éducation constitue le premier ou le deuxième poste budgétaire du gouvernement et souvent la corruption affecte l'allocation des ressources.

Les problématiques sur les dépenses d'éducation ont toujours constitué un terreau fertile pour de nombreux chercheurs dans le monde. Beaucoup de travaux de recherche sur la croissance endogène ont abordé les relations entre les dépenses d'éducation, accumulation de capital humain et la croissance économique à long terme (Lucas, 1988 ; Mankiw et al., 1992 ; Romer, 1990 ; Siggel, 2000 et 2001, etc.). Selon Diebolt (2000), d'une part, les dépenses d'éducation ont un impact sur la croissance économique et, d'autre part, l'éducation est elle-même un investissement soutenu par la croissance. Blankenau et al. (2004 et 2007) ont montré dans une étude empirique que la relation entre les dépenses publiques d'éducation et la croissance n'est pas monotone. Elle dépend du niveau des dépenses publiques, de la structure

\footnotetext{
${ }^{1}$ Les trois objectifs quantifiables de l'Éducation Pour Tous (EPT) sont : l'éducation primaire universelle, la parité entre sexe et l'alphabétisation.
} 
fiscale et des paramètres des technologies de production. Edeh et al. (2018) considèrent, sur la période 1999-2017, que les dépenses d'éducation n'ont pas d'effets significatifs sur le niveau de la pauvreté au Nigéria. Touré D. (2020) montre que l'enseignement supérieur ivoirien a un effet négatif et significatif sur la croissance économique aussi bien à court terme qu'à long terme. Par ailleurs, l'étude réalisée par Reinikka et Svensson (2004) a montré de facto que, dans certains pays en développement, il existe un lien positif entre le niveau élevé de la corruption et les échecs des politiques publiques d'éducation. Cependant, force est de constater que la plupart des modèles où la croissance économique est alimentée par les dépenses publiques d'éducation, une relation non monotone est trouvée entre les deux variables. Mais, aussi, le fait que les dépenses publiques d'éducation agissent positivement sur la croissance économie, tandis que les impôts la ralentissent, constitue toujours une zone d'ombre. Toutefois, le principe de l'équivalence ricardienne stipule que l'impôt et le déficit public génèrent les mêmes conséquences économiques, neutralisant de fait la relance escomptée d'un programme de dépenses publiques. Par ailleurs, les études empiriques sur les effets des dépenses publiques d'éducation sur la croissance économique ne contrôlent pas, de façon explicite, le mode de financement et les canaux de transmission de la corruption.

C'est la raison pour laquelle en connaissant le poids que représente la corruption dans les administrations publiques des pays en développement, il devient stratégique d'intégrer cette variable dans l'analyse des effets des dépenses publiques d'éducation et de la fiscalité sur la croissance économique afin de mieux comprendre les échecs des politiques publiques d'éducation. Les pays d'Afrique subsaharienne regroupent relativement les mêmes caractéristiques en matière de résultats et de politique d'éducation : un faible niveau de leur stock de capital humain et de scolarisation, un taux élevé d'analphabétisme, une faible proportion des ressources allouées à l'éducation, des dépenses publiques d'éducation inefficaces. De ce fait, l'objectif de cet article est d'étudier, à partir de modèles économétriques des données de panel de dix (10) pays d'Afrique de l'ouest sur la période 2004-2020, les effets des dépenses publiques d'éducation et de la fiscalité sur la croissance économique à partir d'un modèle théorique où, d'un côté, ces dépenses jouent un rôle pivot et, de l'autre, la pression fiscale pourrait créer des distorsions dans l'affectation des ressources.

La suite de l'article s'organise de la manière suivante. D'abord, la section 01 expose la revue empirique de la relation entre les dépenses publiques d'éducation, la fiscalité et la croissance économique. Puis, la section 02 traduit une analyse descriptive des systèmes d'éducation, du financement interne de l'éducation et des effets de la corruption en Afrique occidentale. Par la suite, la section 03 met en exergue la spécification du modèle 
économétrique. Enfin, la section 04 aborde les estimations du modèle, l'interprétation des résultats et les discussions.

\section{La revue empirique de la relation dépenses publiques d'éducation, fiscalité et croissance économie}

Elle retient principalement deux approches, l'une purement économétrique et l'autre basée sur des modèles d'équilibre général. Les travaux de Charlot (1997) analysent les liens entre éducation et croissance d'un panel de 125 pays sur la période de 1970-1990. Dans son approche, elle construit une typologie de pays en fonction de l'efficacité de leur système éducatif. Les résultats indiquent que le niveau d'éducation influence positivement le revenu par tête. En effet, la relation entre l'éducation et la croissance économique dépend à la fois du niveau initial de développement du pays et de la qualité de son système éducatif. Autrement dit, dans les pays développés, il faut un certain niveau d'études (apparemment trois années d'études) pour que l'éducation ait des rendements croissants. Alors que dans les pays sous-développés, les niveaux d'éducation et de développement économique sont insuffisants pour créer les conditions d'une mise en valeur du capital humain. Cette analyse constitue un argument de taille visant à inciter les pays en développement à investir davantage dans l'éducation. Dans un autre registre, Herrera (1998) étudie la relation des dépenses publiques d'éducation et la croissance économique en utilisant un modèle convexe d'équilibre général. Les résultats de leurs estimations valident l'existence d'un impact positif des dépenses publiques d'éducation sur la croissance économique à long terme. Blankenau W. F. and Simpson S. B. (2004), en utilisant la méthode d'équilibre général calculable, montrent que la relation des dépenses publiques d'éducation avec la croissance dépend du niveau de ces dépenses, de la structure fiscale et des paramètres des technologies de production. Blankenau W. F. (2005) analyse les effets des dépenses publiques d'éducation dans l'enseignement primaire, secondaire et postsecondaire sur la croissance économique. Il considère que le gouvernement utilise les recettes fiscales pour assurer la qualité de l'enseignement de la maternelle au collège et pour subventionner les frais de scolarité à l'université. Cette subvention n'est possible que si les dépenses publiques d'éducation dans l'enseignement primaire et secondaire dépassent une certaine valeur critique. Ainsi, puisque le capital humain est accumulé grâce à l'enseignement obligatoire de la maternelle au collège, l'augmentation des dépenses d'éducation pourrait avoir effet sur la croissance que lorsqu'une plus grande partie de ces dépenses subventionne les frais de scolarité à l'université. Blankenau et al. (2007) montrent qu'il existe une relation positive entre dépenses publiques d'éducation et croissance économique des pays développés. Cependant, ce lien est sensible aux effets négatifs d'une forte et croissante pression fiscale. 
Les distorsions que pourrait engendrer la pression fiscale peuvent créer des incidences sur l'affectation des ressources. Decaluwé et Maisonnave (2010), en utilisant la méthode d'équilibre général calculable en dynamique séquentielle, analysent à la fois l'impact d'une réforme pro-éducation sur les ménages sud-africains et sur le marché du travail. Leur modèle prend en compte, d'une part, les spécificités du marché du travail en fonction du niveau de qualification (non qualifié, moyennement qualifié, qualifié) et de la race (African, Coloured, Indian et White) et, d'autre part, le comportement des étudiants (abandon, réussite, redoublement). Leurs résultats montrent qu'à court terme, une augmentation des dépenses publiques d'éducation entraînerait une amélioration du comportement des étudiants qui redoublent et abandonnent sans que les revenus des ménages augmentent. Alors qu'à long terme, l'offre de travail qualifié augmenterait de manière inadéquate et finirait par créer du chômage. Cette situation pousserait le gouvernement à chercher à augmenter ses revenus en introduisant une taxe supplémentaire sur le revenu des ménages pour financer la politique éducative. Ce qui entraînerait une détérioration de la consommation des ménages et une baisse de l'activité économique. Coulibaly M. (2013) étudie l'impact des dépenses publiques d'éducation sur la croissance économique en Côte-d'ivoire à partir d'un modèle à correction d'erreurs sur des données de la période 1970-2005. Ses résultats montrent qu'une augmentation de $1 \%$ des taux de croissance de la FBCF et des dépenses d'éducation entraînerait des hausses respectives de $0,17 \%$ et de $5,8 \%$ du taux de croissance économique. Sow (2013), en utilisant la méthode des moindres carrés ordinaires (MCO) et l'approche par la frontière stochastique de production, a abouti aux résultats selon lesquels l'éducation produit un effet positif sur la croissance économique du Sénégal, à travers l'impact positif des niveaux d'études du chef d'entreprise et du salarié sur la productivité. Edeh, Obi et Obi (2018) ont estimé les effets du stock de capital, de la scolarisation primaire et des dépenses d'éducation dans la réduction de la pauvreté au Nigéria à partir des MCO sur la période 19992017. Les résultats de leur estimation montrent que les dépenses d'éducation n'ont pas un effet significatif sur le niveau de pauvreté. Touré D. (2020), en utilisant la méthode d'estimation des variables autorégressives à retards échelonnés sur la période 1970-2016, souligne que l'enseignement supérieur ivoirien affecte négativement et significativement la croissance économique aussi bien à court terme qu'à long terme.

\section{Analyse des secteurs éducatifs en Afrique de l'ouest de 2000-2020}

En Afrique de l'ouest, les systèmes éducatifs sont régis par des lois et composés de deux identités : l'une concerne l'enseignement public et l'autre l'enseignement privé qui peut être laïc ou confessionnel (catholique, protestant et islamique). Ils sont aussi composés de six (6) niveaux: l'éducation 
préscolaire, l'enseignement primaire, l'enseignement moyen et secondaire général, l'enseignement technique et la formation professionnelle et l'enseignement supérieur. À cela, s'ajoute le système non formel ${ }^{2}$ qui comprend les centres permanents d'alphabétisation et de formation, les centres d'éducation de base non formelle, etc.

Dans les pays anglophones comme le Ghana et la Gambie, les enfants entament leurs études à l'âge de 3 ou 4 ans, allant de la prématernelle à la maternelle, puis, à l'école primaire qui dure six (6) ans, puis, au premier cycle ( 3 ans), puis, au deuxième cycle (3 ans) de l'enseignement secondaire et, enfin, le cycle universitaire (un cursus de 4 ans). Parallèlement, dans les pays francophones tels que le Bénin, le Burkina-Faso, la Côte-d'Ivoire, le Mali, le Niger, le Sénégal et le Togo, le système éducatif commence par le préscolaire, puis l'enseignement primaire qui dure six (6) ans, puis, le moyen (4 ans) et le secondaire général ( 3 ans) et, enfin, le supérieur ( 5 ans). Dans les pays lusophones comme le Cap-Vert, le système éducatif formel comprend l'éducation préscolaire, l'enseignement primaire qui dure six (6) ans, le secondaire ( 6 ans) et le supérieur ( 4 ans). En plus, le système intègre aussi une composante extrascolaire vouée à l'éducation des adultes (à partir de 15 ans), articulant une formation générale de base (en trois phases) et une formation professionnelle (Ferreira, 2013).

\subsection{Une description du niveau de la scolarisation en Afrique de l'ouest}

En 1960, le taux de scolarisation des classes d'âge 6-11 ans n'était que de $30 \%$. Au début des années 2000 , le seuil des $80 \%$ est atteint dans plusieurs pays d'Afrique subsaharienne, soit un bond de 50\% sur quatre décennies. De 2000 à 2020, les meilleurs taux moyens de scolarisation à l'école primaire en Afrique de l'ouest sont réalisés par le Togo (121\%), le Bénin $(113,7 \%)$ et le Cap-Vert $(110,8 \%)$. Toutefois, la valeur théorique maximale du taux net de scolarisation au primaire (TNSP) est de 100\%. Le TNSP dans le monde est passé de $83,4 \%$ à $89,8 \%$ entre 2000 et 2019. Dans la figure $\mathrm{n}^{\circ} 01$, nous observons que de 2009 à 2020 le Bénin $(95,9 \%)$, le Cap-Vert $(93,4 \%)$ et le Togo $(90,6 \%)$ enregistrent les TNSP moyens les plus élevés, contrairement le Mali et le Niger qui se situent respectivement à $60,7 \%$ et $60,5 \%$. Durant la même période, le Cap-Vert $(93,4 \%)$ enregistre le taux moyen d'achèvement aux études primaires le plus élevé, suivi du Ghana $(92,4 \%)$ et le Togo $(81,7 \%)$. Le Niger $(54,4 \%)$ et le Mali $(53,1 \%)$ enregistrent les taux moyens d'achèvement aux études primaires les plus bas. De 2000 à 2020, c'est le CapVert, le Béenin, le Ghana et le Togo qui réussissent à réaliser les meilleures performances de scolarisation des enfants à l'école primaire.

\footnotetext{
${ }^{2}$ Le système d'éducation non formel concerne toutes les activités d'éducation et de formation structurées et organisées dans un cadre non scolaire.
} 


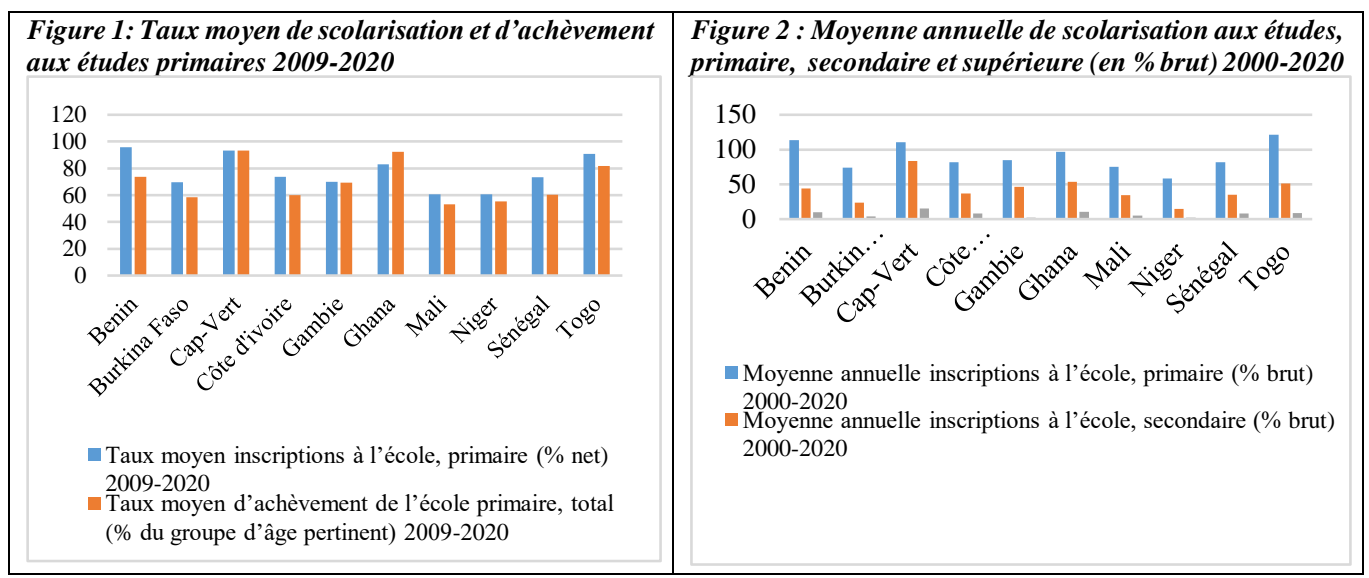

Source : Données Banque Mondiale et UNESCO

Les taux moyens de scolarisation aux études secondaires et supérieures sont plus élevés au Cap-Vert (respectivement 83,7\% et 15,3\%) et au Ghana $(53,5 \%$ et $10,5 \%)$. Cependant, le Burkina-Faso (respectivement $23,9 \%$ et $3,84 \%$ ) et le Niger (14,6\% et $2,03 \%$ ) réalisent les taux moyens de scolarisation aux études secondaires et supérieures les plus bas. Toutefois, la moyenne des taux de scolarisation aux études supérieures reste très faible. Selon Spence (1973), l'investissement dans le capital humain est pour l'individu un moyen de signaler ses capacités aux entreprises. De ce fait, en termes de coût d'opportunité, seuls les individus les plus productifs trouveront rentable de réaliser cet investissement, car ils s'attendent à percevoir des salaires futurs élevés pouvant compenser les revenus perdus aujourd'hui. Malheureusement, cet investissement éducatif est une contrainte majeure dans les pays de l'Afrique de l'ouest, la grande partie de la population n'a pas cette opportunité. Cette faiblesse du taux de scolarisation aux études supérieures ralentit la diffusion du progrès technique. Cela limite la demande de nouvelles compétences. Ce qui pourrait remettre en cause l'utilité même de l'investissement éducatif à rendre dynamique le marché du travail.

\subsection{Le coût de l'éducation en Afrique de l'ouest : un état des lieux}

Les niveaux des dépenses publiques d'éducation sont certes insuffisants, mais ils connaissent une progression dans le temps presque partout en Afrique. À cela, s'ajoute la contribution massive des ménages au financement de l'éducation (en moyenne $46 \%$ des dépenses publiques par élève selon UNESCO, 2012). Certes, aujourd'hui, en Afrique de l'ouest, les gouvernements mobilisent plusieurs stratégies afin d'améliorer le financement de l'éducation. Mais, cela ne cache pas les fortes disparités existant entre les dépenses par élève du secondaire et celles de l'enseignement supérieur. Alors 
que, dans les pays d'Amérique du Nord et d'Europe, les dépenses par élève dans le primaire sont équivalentes à celles de l'enseignement supérieur.

La figure $\mathrm{n}^{\circ} 03$ nous renseigne qu'en 2000, au Cap-Vert, les dépenses par élève de l'enseignement supérieur par rapport au PIB par habitant font 13,8 fois celles du secondaire, au Togo 12,6 fois, au Niger 12,5 fois et la Côted'Ivoire 3,4 fois. En 2019, les dépenses par élève de l'enseignement supérieur font 16,68 fois celles du secondaire au Niger, 11,9 fois au Sénégal, 11 fois au Burkina-Faso et 1,8 fois au Cap-Vert. Aujourd'hui, le nivellement est en train de s'opérer de plus en plus dans certains pays de l'Afrique de l'ouest, c'est le cas du Cap-Vert, du Ghana, du Togo et du Bénin.

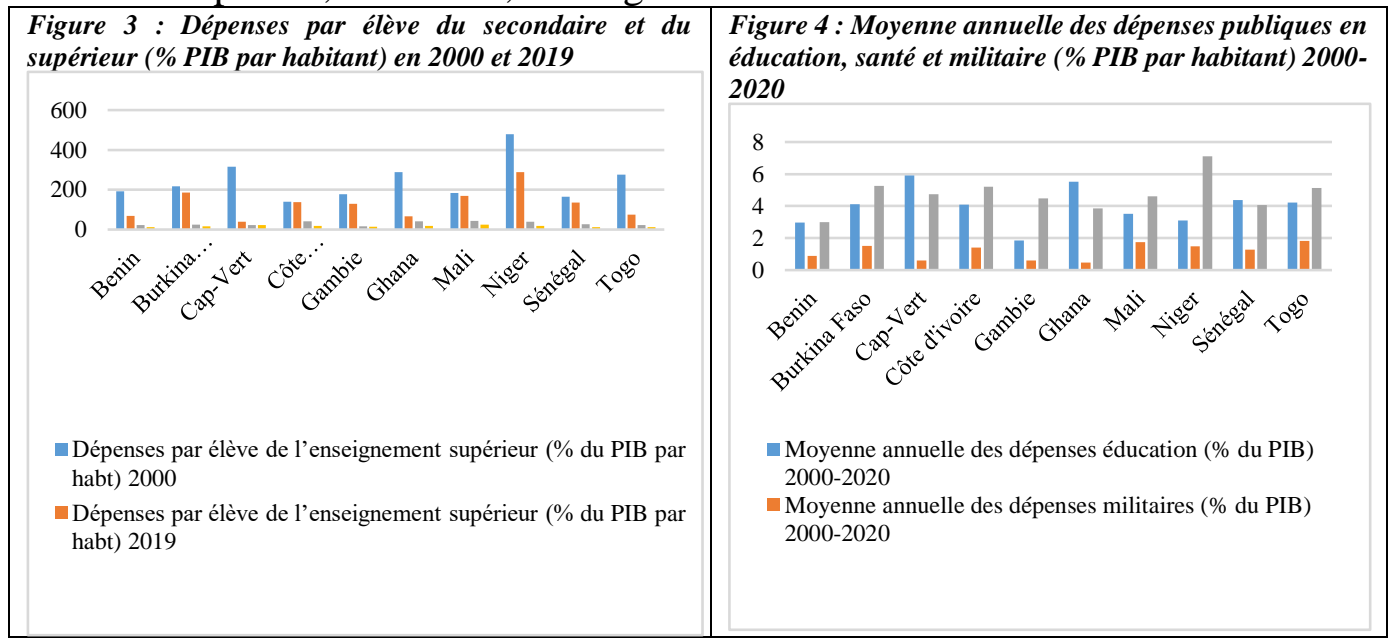

Source : Données Banque Mondiale et UNESCO

La figure $n^{\circ} 04$ nous renseigne sur les choix des priorités entre dépenses publiques en éducation, en santé et militaires. Au moment où les gouvernements cap-verdiens (avec 5,9\% du PIB) et ghanéens (5,5\% du PIB) rendent prioritaires les dépenses publiques d'éducation sur les dépenses de santé (respectivement $4,7 \%$ et $3,9 \%$ du PIB) et militaires $(0,6 \%$ et $0,5 \%$ du PIB), le Niger et le Burkina-Faso optent davantage pour l'orientation des dépenses publiques vers la santé (respectivement $7,1 \%$ et $5,3 \%$ du PIB) et la défense (1,5\% et 1,45\%). Cela peut s'expliquer par le fait que depuis le début des années 2000, les gouvernements malien, burkinabe et nigérien mobilisent beaucoup de moyens financiers pour se réarmer afin de mieux faire face aux menaces perpétrées par la rébellion touareg et des groupes djihadistes liés à Al-Qaïda. Aujourd'hui, la pandémie de COVID-19 crée des chocs entrainant une baisse de la demande et de l'offre d'éducation dans la plupart des pays d'Afrique subsahariens. En effet, la baisse de la demande d'éducation s'est traduite surtout par une hausse des taux d'abandon des élèves défavorisés et une diminution de l'investissement des parents dans l'éducation. L'offre d'éducation suit la même tendance, elle est, d'une part, marquée par la baisse 
des dépenses publiques d'éducation et de la qualité de l'enseignement et, d'autre part, la fermeture de plusieurs écoles privées à cause d'insuffisances de ressources financières d'exploitation.

\subsection{Relation dépenses publiques d'éducation, recettes fiscales et corruption}

Plus l'indice de perception de la corruption est élevé, plus le niveau de corruption dans le pays est faible.

Tableau 01 : Corrélation entre corruption, croissance économique, recettes fiscales, dépenses publiques d'éducation et dépenses publiques hors éducation d'un panel de 10 pays d'Afrique de

\begin{tabular}{|l|l|l|l|l|}
\hline & $\begin{array}{l}\text { Croissance } \\
\text { économique }\end{array}$ & $\begin{array}{l}\text { Recettes } \\
\text { fiscales }\end{array}$ & $\begin{array}{l}\text { Dépenses } \\
\text { publiques } \\
\text { d'éducation }\end{array}$ & $\begin{array}{l}\text { Dépenses publiques } \\
\text { hors éducation }\end{array}$ \\
\hline Corruption & $0,1998^{*}$ & $0,5353^{*}$ & $0,6231^{*}$ & $0,5210^{*}$ \\
\hline \multicolumn{7}{|c|}{ Seuil de significativité $(*=5 \%)$} \\
\hline
\end{tabular}

Source : Calculs de l'auteur

Le tableau $\mathrm{n}^{\circ} 01$ nous renseigne qu'il existe une faible corrélation positive entre l'indice de perception de la corruption et la croissance économique. Selon Hysa (2011), il existe une relation négative et statistiquement significative entre la corruption et des composantes du développement humain telles que la santé, l'éducation et le revenu. Dans une autre étude menée par Murshed et Mredula (2018), la corruption est considérée comme un facteur pouvant retarder la réalisation des objectifs du millénaire pour le développement en 2030 à travers le monde. Honlonkou A. (2003) considère que la corruption affecte la croissance économique à partir d'un certain niveau de développement. Selon lui, la corruption n'entrave pas la croissance économique globale des pays qui ont une économie extravertie et qui est caractérisée par un sous-emploi des facteurs de production et la prédominance des secteurs de rentes. Enfin, nos résultats montrent l'existence d'une corrélation positive entre l'indice de perception de la corruption et les recettes fiscales. En effet, la corruption entrave la capacité du gouvernement à collecter l'impôt.

\section{Modèle économétrique}

\subsection{Modèle théorique}

Pour cette étude, nous nous sommes référé au modèle théorique de BST (William F. B., Simpson, N. B. and Tomljanovich M., 2007). De fait, ce modèle prend en compte trois dimensions temporelles : la période de la naissance de l'agent, celle de sa jeunesse et enfin la période où il devient un travailleur. Autrement dit, d'abord, nous considérons que l'agent $m$ est né à chaque période, puis, à la période $t$, il devient jeune et reçoit de la part du 
gouvernement une dotation correspondant au paiement des frais d'éducation publique $F E_{t}$ et, enfin, ces contributions publiques se combinent avec le capital humain de la génération précédente $H_{t}$, pour créer du capital humain, en tant que travailleur à partir de l'équation $\mathrm{n}^{\circ} 01$.

$$
H_{t+1}=\delta F E_{t}^{\mu} h_{t}^{1-\mu} ; \mu \in[0,1], \delta>0
$$

L'agent devenant travailleur à la période $t+1$, en évidence, reçoit un revenu net égal à $w_{t+1} H_{t+1}\left(1+\tau_{m}\right)$, où $\tau_{m}$ est le taux d'imposition sur le revenu et $w$ représente le salaire d'une unité de capital humain. Le travailleur épargne pour sa retraite en accumulant du capital.

En effet, l'accumulation du capital à la fin de la période $t+1$ est $K_{t+2}$. Une unité de capital placée à la période $t+1$ génère une rente $r_{t+2}\left(1-\tau_{m}\right)$ unités à la période $t+2$, où $r$ représente le taux de capitalisation.

Alors, à la période $t$, l'agent maximise sa fonction d'utilité à vie en choisissant :

- de consommer $C_{t, t+1}$ à la période $t+1$ et $C_{t, t+2}$ à la période $t+2$;

- d'accumuler du capital pour la vieillesse $K_{t+2}$ à la fin de la période $t+$ 1.

La fonction d'utilité est un logarithme des consommations $C_{t, t+1}$ et $C_{t, t+2}$ et elle est actualisée à la période $t+2$ au taux $\beta$. En plus, la consommation est imposable au taux $\tau_{c}$.

La contrainte budgétaire de l'agent :

- à la période $t+1$ est $C_{t, t+1}\left(1+\tau_{c}\right)+K_{t+2} \leq w_{t+1} H_{t+1}\left(1-\tau_{m}\right)$;

- à la période $t+2$ est $C_{t, t+2}\left(1+\tau_{c}\right) \leq r_{t+2}\left(1-\tau_{m}\right) K_{t+2}$.

La solution optimale au problème de maximisation de la fonction d'utilité dégage un niveau optimal d'accumulation du capital pour la vieillesse,

$$
K_{t+2}=\beta(1+\beta)^{-1} w_{t+1} H_{t+1}\left(1-\tau_{m}\right)
$$

Toute firme performante cherche à combiner le capital physique avec le capital humain afin de réaliser une production de grande qualité et très compétitive $y_{t}=A k_{t}^{\alpha}$ où $y$ est l'output par unité de capital humain et $k$ est le ratio entre le capital physique et le capital humain.

Les paramètres d'ajustement sont $\alpha \in[0,1]$ et $A>0$. Ainsi, le programme d'optimisation de la firme est $r_{t}=A \alpha k_{t}^{\alpha-1}$ et $w_{t}=A(1-$ $\alpha) k_{t}^{\alpha}$.

Par ailleurs, le gouvernement consacre une part Dpe du budget de l'État aux dépenses d'éducation. Le reste du budget est consacré aux dépenses 
autres que celles d'éducation $D p h e$ (par exemple, dépenses santé, militaires, etc.).

Les dépenses publiques sont financées par les impôts sur le revenu du travail et de la propriété, par les taxes à la consommation ou par des emprunts publics correspondant aux dettes extérieures Dtpex, évaluées par rapport au niveau de la production $Y_{t}$.

Alors, les politiques publiques sont définies $\left\{\tau_{m}, \tau_{c}, D p e, D p h e, D t p e x\right\}$ et elles sont sous contrainte budgétaire selon l'équation (03).

$$
\tau_{m} w_{t} H_{t}+\tau_{m} r_{t} K_{t}+\tau_{c}\left(C_{t-1, t}+C_{t-2, t}\right)=(\text { Dpe }+ \text { Dphe }+ \text { Dtpex })
$$

Car les dépenses publiques d'éducation sont liées aux frais d'éducation par la relation $F E_{t}=\widetilde{D p e} Y_{t}$, où $\widetilde{D p e}=\exp (D p e)$.

L'équilibre du taux de croissance économique à long terme transforme l'équation (01) par la relation suivante :

$$
1+G P D=\rho\left(\widetilde{D p} e A k^{\alpha}\right)^{\mu}
$$

Où $G P D$ est le taux de croissance économique.

La substitution des équations (01) et les salaires dans l'équation (02) permet d'établir l'expression suivante : $k=k\left(\rho, A, \alpha, \beta, \mu, D p e, \tau_{m}\right)$. Maintenant, l'intégration de l'expression de $k$ dans l'équation (04), en utilisant le logarithme et la formule du développement limité $\ln (1+x) \approx x$, donne

$$
G P D=\underline{\beta_{0}}+\beta_{1} D p e+\beta_{2} \tau_{m}
$$

Où $\underline{\beta_{0}}=\underline{\beta_{0}}(A, \rho, \alpha, \beta, \mu), \beta_{1}=\beta_{1}(\alpha, \mu)$, et $\beta_{2}=\beta_{2}(\alpha, \mu)$.

Enfin, pour compléter le modèle, nous utilisons l'équation (03) afin d'établir la relation entre $D p e$ et $\tau_{m}$. Les recettes fiscales financent en partie les dépenses publiques.

Soit $\widetilde{C}_{t}$ représentant la consommation totale.

Par conséquent, l'équation (03) implique $\tau_{m}=\frac{(D p e+D p h e+D t p e x)}{(1+\varphi)}$; où $\left.\varphi=\frac{\left(\tau_{c} \widetilde{C_{t}}\right.}{\left(\tau_{m} Y_{t}\right.}\right)$ est le rapport entre les recettes fiscales liées à la consommation et le total des recettes fiscales. Ce ratio est constant en situation de croissance économique d'équilibre.

L'équation (05) devient GPD $=\underline{\beta_{0}}+\beta_{1} D p e+\beta_{2} \frac{(\text { Dpe+Dphe+Dtpex })}{(1+\varphi)}$.

\subsection{Spécification du modèle et des variables}

Pour passer à la spécification du modèle, nous supposons d'abord que $\underline{\beta_{0}} \approx \beta_{0}$ est une constante et $n$ le nombre d'observations. Également, nous 
allons chercher à contrôler, dans le temps, l'hétérogénéité pour chaque pays. Avec $\varrho_{i}$, un effet fixe dans le temps, mais qui est différent pour chaque pays, $\delta_{t}$, un effet temporel commun à tous les pays et différent suivant les années, $u_{i, t}$, le terme d'erreurs, constituant les perturbations aléatoires i.i.d N $\left(0, \sigma_{u}^{2}\right)$ et non auto-corrélées. Ainsi, la régression de la croissance économique devient,

$$
\begin{gathered}
G P D_{i, t}=\beta_{0}+\beta_{1} \text { Dpe }_{i, t-5}+\beta_{2}\left[\frac{\text { Dpe }_{i, t}+\text { Dphe }_{i, t}+\text { Dtpex }_{i, t}}{1+\varphi_{i, t}}\right]+\beta_{3} T s p_{i, t} \\
+\beta_{4} \operatorname{Tsec}_{i, t}+\beta_{5} \operatorname{Tsup}_{i, t}+\beta_{6} I P C_{i, t}+\varrho_{i}+\delta_{t}+u_{i, t}
\end{gathered}
$$

$G P D$, est la variable dépendante. Elle représente pour chaque pays le taux de croissance du PIB réel annuel par tête à la période $t$.

Á côte, une batterie de variables indépendantes entre en jeu.

Dpe, ce sont les dépenses consacrées à l'éducation publique en plus des subventions accordées aux écoles privées du niveau primaire, secondaire et tertiaire, en pourcentage du PIB à la période $t-5$. Les dépenses publiques d'éducation doivent accuser un retard de cinq (05) ans, car il faut du temps pour qu'elles puissent avoir un impact sur la croissance économique.

$\tau$, il s'agit de la pression fiscale à la période $t$. Elle correspond au montant total des recettes fiscales recouvrées, elle est exprimée en pourcentage du PIB. Selon Blankenau W. et al. (2007), si les effets d'éviction ne sont pas maîtrisés (c'est-à-dire que les dépenses publiques d'éducation soient très élevées par rapport aux investissements et consommations du privé dans le secteur), les distorsions engendrées par la pression fiscale peuvent modifier les effets positifs des dépenses publiques d'éducation sur la croissance ;

Dphe, Dépenses publiques hors éducation en pourcentage du PIB à la période $t$.

Dtpex, il s'agit des dettes extérieures contractées dans l'année ou garanties par le secteur public en pourcentage du PIB à la période $t$. Il est important de signaler que dans les pays d'Afrique de l'ouest, la dette intérieure est très faible par rapport à celle de l'extérieur. Par exemple, au Sénégal, la dette extérieure 2020 représente près de $89 \%$ du total de la dette publique).

Tasp, Tsec et Tsup sont respectivement les taux bruts d'achèvement de l'école primaire, de scolarisation aux études secondaires et supérieures en pourcentage de la population totale du pays $i$ à la période $t$.

$I P C$ est l'indice de perception de la corruption du pays $i$ à la période $t$. Plus il est faible, plus le niveau de corruption est élevé. 


\section{Analyse économétrique et discussion des résultats \\ 4.1. Analyse économétrique des données}

Notre objectif principal est de réaliser une série de régressions sur données de panel par la méthode des moindres carrés ordinaires (MCO) dans lesquels nous analysons les effets des dépenses publiques d'éducation sur la croissance économique. Les données utilisées proviennent des bases de données de la Banque Mondiale et de l'UNESCO. Pour toutes les variables, nous utilisons des données annuelles couvrant la période 2004-2020 de 10 pays d'Afrique occidentale, sauf celles des dépenses publiques d'éducation qui accusent un retard de 5 ans, donc étudiées sur la période allant de 1999 à 2016. Les dépenses publiques d'éducation sont financées par les ressources publiques internes et les aides et dettes extérieures. Par ailleurs, il est important de signaler que Blankenau W. et al. (2007) ont intégré dans leur modèle le niveau initial de développement qui est souvent considéré comme ayant un impact sur la vitesse de croissance économique des pays (Barro et Sala-iMartin, 1999). De notre part, nous considérons que le niveau initial de développement n'est pas pertinent dans notre modèle spécifique car tous les pays de notre échantillon ont toujours été pauvres et très endettés.

Pour le tableau 02, nous avons réalisé des tests de robustesse afin de corriger les problèmes d'autocorrélation des erreurs et d'hétéroscédasticité dans les régressions (1), (2) et (3). En effet, ils testent l'influence de chaque pays sur la significativité des résultats des régressions. Sur les trois régressions, ils valident le non rejet de l'hypothèse $H o$. Les résultats des régressions sont interprétés séparément.

Tableau 2 : Série d'estimations MCO des effets des dépenses publiques d'éducation sur la croissance économique

\begin{tabular}{|c|c|c|c|c|c|}
\hline Régression & $(1)$ & $(2)$ & $(3)$ & BST & KBG \\
\hline Dpe -5 & $* * 0.4603$ & $* 0.3467$ & $* * 0.442$ & $* 0.201$ & 0.128 \\
& $(0.0292)$ & $(0.189)$ & $(0.196)$ & $(0.144)$ & $(0.110)$ \\
\hline$\tau$ & - & -0.07389 & -0.02367 & $* *_{-} 0.099$ & -0.029 \\
& & $(0.0771)$ & $(0.077)$ & $(0.044)$ & $(0.031)$ \\
\hline Dphe & - & -0.05487 & -0.0682 & 0.0002 & -0.027 \\
& & $(0.0485)$ & $(0.0487)$ & $(0.038)$ & $(0.037)$ \\
\hline Dtpex & - & $* *-0.0369$ & $* *-0.0368$ & $* * 0.128$ & $* * 0.087$ \\
& & $(0.0077)$ & $(0.0085)$ & $(0.042)$ & $(0.039)$ \\
\hline Tspr & - & - & -0.0159 & -0.022 & -0.020 \\
& & & $(0.027)$ & $(0.018)$ & $(0.018)$ \\
\hline Tsec & - & - & -0.00175 & & \\
& & & $(0.0323)$ & & \\
\hline Tsup & - & - & $* *-0.2192$ & & \\
\hline IPC & - & $* 0.0591$ & $* * 0.1124$ & & \\
\hline Ajusted $R^{2}$ & 0.105 & 0.173 & 0.241 & 0.266 & 0.243 \\
& 170 & 170 & 170 & 137 & 137 \\
\hline
\end{tabular}


\begin{tabular}{|c|c|c|c|c|c|}
\hline & $E A$ & $E A$ & $E A$ & & \\
\hline
\end{tabular} Blankenau, Simpson, and Tomljanovich (2007) ; KBG = Kneller, Bleaney, and Gemmell (1999).

Source : Calculs de l'auteur

D'abord, dans la régression $(01)$ du tableau $n^{\circ} 02$, nous cherchons à établir uniquement une relation basique entre les dépenses publiques d'éducation et la croissance économique par habitant. Pour cela, nous avons introduit dans la régression des effets fixes dans le temps et qui sont différents pour chaque pays. En plus, non seulement nous faisons abstraction de la manière dont l'éducation est financée, mais nous considérons que les dépenses publiques d'éducation réalisées à la date $t$ n'ont un effet sur la croissance économique qu'à partir de la date $t+5$. Nos résultats montrent que les dépenses publiques d'éducation $D p e_{-5}$ ont un effet marginal positif et significatif sur la croissance économique par habitant à long terme, comme l'indiquent ceux de Blankenau et al. (2007).

Puis, dans la régression (02), nous analysons les effets de certaines composantes du budget des gouvernements sur la croissance économique par habitant. À cet effet, nous considérons que les dépenses publiques d'éducation sont financées à la fois par les recettes fiscales et la dette extérieure. Nos résultats montrent que les dépenses publiques d'éducation $D p e_{-5}$ ont un effet marginal positif et significatif sur la croissance économique par habitant (une augmentation de $1 \%$ des dépenses publiques d'éducation s'est traduite par un accroissement de 0,35 point de croissance).

Les recettes fiscales $\tau$, qui financent une partie des dépenses d'éducation, ont un effet marginal négatif comme l'évoquent les résultats de Blankenau et $a l$. (2007) et Kneller et al. (1999). Cependant, leur impact sur la croissance économique par habitant n'est pas significatif. Donc, la relation entre les dépenses publiques d'éducation et la croissance économique n'est pas sensible à la pression fiscale car les dépenses des ménages à l'éducation en Afrique subsaharienne représentent $46 \%$ des dépenses d'éducation faites par les États (1'UNESCO ${ }^{3}, 2012$ ).

Comme les résultats de Kneller et al. (1999), les dépenses publiques hors éducation $D p h e$ (constituées de dépenses de santé et militaires) ont un effet marginal négatif et non significatif sur la croissance économique par habitant. Certes, les résultats de Blankenau et al. (2007) et Kneller et al. (1999) valident l'effet positif et significatif de la dette publique sur la croissance dans les pays développés. Mais, nos résultats montrent le contraire, puisque les

\footnotetext{
${ }^{3}$ Foko B., Tiyab K. B., Husson G. (2012), «Les Dépenses des ménages en éducation : une perspective analytique et comparative pour 15 pays d'Afrique ». UNESCO Office Dakar and Regional Bureau for Education in Africa.
} 
dettes extérieures Dtpex représentant la quasi-totalité des dettes publiques des pays de notre échantillon ont un effet marginal négatif et significatif sur la croissance.

L'indice de perception de la corruption IPC exerce un effet marginal positif et significatif sur la croissance économique par habitant.

Enfin, la particularité de la régression (03) est qu'en plus des composantes budgétaires et de la corruption, elle tient compte des effets des taux de scolarisation des différents niveaux d'études sur la croissance économique. Également, nous avons inclus dans la régression des effets temporels communs à tous les pays. Comme dans les régressions (01) et (02), dans la régression (03), les dépenses publiques d'éducation $D p e_{-5}$ exercent un effet positif et significatif sur la croissance.

Nos estimations dans la régression (03) révèlent que les taux d'achèvement à l'école primaire et de scolarisation aux études du secondaire n'ont pas d'effets significatifs sur la croissance économique. Par contre, le taux de scolarisation aux études supérieures a un effet négatif (ce qui est normal, car le capital humain comme le capital physique sont des inputs qu'on transforme pour créer de la production) et significatif sur la croissance économique.

\subsection{Discussion des résultats d'étude}

Cette étude atteste d'abord la contribution significativement positive des dépenses publiques déducation au processus de croissance économique à long terme, comme l'indiquent les travaux de Blankenau et al. (2007). Puis, elle met l'accent sur le mode de financement de l'éducation publique. Á l'image des résultats de Blankenau et al. (2007) et Kneller et al. (1999), les recettes fiscales et les dettes publiques ont un effet marginal négatif sur la croissance économique. Enfin, elle vérifie que l'indice de perception de la corruption exerce un effet marginal positif et significatif sur la croissance économique.

Cette étude confirme donc les résultats de Herrera R. (1998), de Decaluwé et Maisonnave (2010) et de Coulibaly M. (2013) selon lesquels les dépenses publiques d'éducation ont un effet positif sur la croissance économique. Cependant, dans une demi-mesure, ces auteurs ne partagent pas le point de vue de Edeh et al. (2018) selon lesquels les dépenses d'éducation n'ont pas un effet significatif sur le niveau de pauvreté au Nigéria.

Les recettes fiscales ont un effet négatif et non significatif sur la croissance économique. En réalité, la non-significativité des recettes fiscales sur la croissance économique peut être expliquée par les faibles niveaux de revenus, même parmi les entreprises et les particuliers officiellement enregistrés, qui réduisent l'assiette sur laquelle reposent les impôts sur le revenu et la consommation. Á cela, s'ajoute le poids élevé du secteur informel. 
En effet, dans les pays africains, le niveau élevé de l'emploi et de l'entreprise informels constitue un obstacle à la mobilisation optimale des recettes fiscales. Ainsi, la relation entre la fiscalité et la croissance économique dépend de la structure de la fiscalité. Pour Johansson et al. (2008), une réforme fiscale sans incidence sur les impôts et orientée vers la croissance consisterait à transférer une partie de la base imposable des impôts sur le revenu, sur des impôts moins générateurs de distorsion, comme les impôts récurrents sur l'immobilier ou ceux sur la consommation.

Cette étude partage les assertions selon lesquelles la dette extérieure constitue un frein à la croissance économique. Elle conforte les allégations de Kumar et Woo (2010) qui considèrent que l'effet négatif de la dette extérieure des pays avancés et émergents sur la croissance économique à long terme reflète en grande partie un ralentissement de la croissance de la productivité du travail principalement dû à une réduction des investissements et à une croissance plus lente du stock de capital. Cependant, elle ne partage pas le point de vue de Blankenau et al. (2007) et Kneller et al. (1999) qui considèrent que le niveau de la dette publique d'un pays a des effets positifs significatifs sur la croissance économique par habitant. En réalité, dans les pays d'Afrique de l'ouest, la dette publique ne pourrait avoir un effet positif et significatif sur la croissance économique que si les gouvernements parvenaient à assurer sa viabilité à moyen et long terme. Mais, si la dette publique est utilisée pour financer des dépenses récurrentes, c'est tout à fait normal que des passifs s'accumulent et, à la longue, les gouvernements pourront avoir beaucoup de mal à assurer sa viabilité. Cependant, si la dette publique est utilisée pour financer des investissements destinés à diversifier l'économie et à valoriser le capital humain, cela permettrait aux gouvernements de pouvoir mettre en place les conditions nécessaires pour créer davantage de richesses, de faire face aux chocs extérieurs imprévisibles et d'acquérir de la capacité de remboursement.

L'indice de perception de la corruption exerce un effet marginal positif et significatif sur la croissance économique. Les pays qui ont les indices de perception de la corruption les plus élevés comme le Cap-Vert (56) et le Ghana (42), c'est-à-dire ceux qui ont les niveaux de corruption les plus bas, sont ceux qui ont réalisé plus de performance économique ces deux dernières décennies et ont dépensé plus dans l'éducation publique, avec des moyennes annuelles respectives de $5,4 \%$ et $5,1 \%$ du PIB. Cette étude conforte les allégations de Hysa (2011) qui considère qu'il existe une relation négative et statistiquement significative entre la corruption et des composantes du développement humain telles que la santé, l'éducation et le revenu. Mieux, elle vient conforter la position de Murshed et Mredula (2018) sur la capacité de la corruption à retarder la réalisation des objectifs du millénaire pour le développement en 2030 à travers le monde. 
Enfin, le taux de scolarisation aux études supérieures a un effet négatif et significatif sur la croissance économique. Cette étude est en phase avec les résultats de Touré D. (2020) à travers lesquels l'enseignement supérieur ivoirien affecte négativement et significativement la croissance économique aussi bien à court qu'à long terme.

Toutes les théories économiques trouvent l'éducation comme un investissement important pour le développement d'un pays. Car, une augmentation du niveau de la scolarisation devrait produire un effet positif sur le capital humain et, par la suite, sur la croissance économique. Mais, en Afrique subsaharienne, le contexte est particulier car l'économie informelle représente entre $25 \%$ et $65 \%$ du PIB et entre $30 \%$ et $90 \%$ de l'emploi non agricole (FMI, 2017). C'est là où se situe le paradoxe, car le PIB est calculé sur la base de l'économie formelle et qui exige un certain niveau de qualité de capital humain, donc un niveau d'études plus élevé, alors que les opérateurs économiques dans le secteur informel, en majorité, ont un niveau d'éducation souvent bas. Par ailleurs, le poids élevé du taux de chômage et la tertiarisation des économies en Afrique de l'ouest font que les plus scolarisés ont plus de chance de trouver un emploi.

\section{Conclusion}

Cette étude analyse les effets des dépenses publiques d'éducation, la fiscalité et la corruption sur la croissance économique à long terme à partir de modèles économétriques des données de panel de 10 pays d'Afrique de l'ouest sur la période 2004-2020. Les résultats de nos estimations montrent qu'il existe une relation positive et significative entre les dépenses publiques d'éducation retardées de 5 ans et la croissance économique par habitant. Le second enseignement nous renvoie aux effets négatifs aussi bien de la fiscalité que de la dette publique sur la croissance économique par habitant. Ce résultat est en adéquation avec le principe de l'équivalence ricardienne. Parallèlement, la corruption, en affectant significativement la croissance économique, entraîne des pertes importantes de recettes fiscales. Enfin, force est de repérer que dans nos estimations, seul le taux de scolarisation aux études supérieures exerce un effet significatif sur la croissance à long terme.

Toutefois, cette étude essaie d'apporter une réponse à certaines problématiques de politique économique. Nos résultats laissent ainsi entrevoir que les dépenses publiques d'éducation améliorent la croissance à long terme dans les pays d'Afrique de l'ouest, tant que les effets d'éviction sont contrôlés, la viabilité de la dette publique est assurée à long terme et la corruption maîtrisée. L'atteinte des objectifs de l'éducation pour tous entraîne inéluctablement l'intervention du secteur public. Par conséquent, cela va augmenter les dépenses publiques. Les gouvernements d'Afrique de l'ouest sont donc invités à mener des actions vigoureuses dans le management des 
dépenses publiques d'éducation et de son financement. Mais, aussi, ils doivent inciter le secteur informel à se formaliser pour développer des capacités à mobiliser des ressources publiques internes afin de prendre en charge, en toute indépendance, les dépenses publiques d'éducation.

Cependant, cette analyse connaît des limites. Elle doit être approfondie au niveau théorique. En effet, cela consiste à plus développer les fondements macro et microéconomiques de la relation entre les dépenses publiques d'éducation, la fiscalité, la corruption et la croissance économique afin de mieux repositionner la robustesse de nos conclusions. En outre, la période de l'étude est relativement courte (2004-2020) et naturellement les résultats des estimations économétriques pourraient en être affectés.

À la lumière du débat actuel sur la bonne gouvernance et les difficultés à mobiliser des ressources publiques en Afrique, nos travaux futurs seront essentiellement influencés par la problématique de la corruption dans le financement des investissements publics.

\section{References:}

1. Barro R. J. and Sala-i-Martin X. (1999). Economic Growth. Cambridge, MA: MIT Press.

2. Blankenau W. F., and Simpson N. B. (2004). Public Education Expenditures and Growth. Journal of Development Economics, 73(2): 583-605.

3. Blankenau W. F. (2005). Public Schooling, College Subsidies and Growth. Journal of Economic Dynamics and Control, 29(3): 487-507.

4. Blankenau W. F., Simpson N. B. and Tomljanovich M. (2007). Public Education Expenditures, Taxation, and Growth: Linking Data to Theory. American Economic Review, 97 (2): 393-397.

5. Charlot S. (1997). La relation éducation-croissance : apports théoriques récents et tests empiriques. Document de travail. Laboratoire d'analyse et de techniques économiques (LATEC), p. 226.

6. Coulibaly M. (2013). Impact des dépenses publiques d'éducation sur la croissance économique en côte d'ivoire. European Scientific Journal, 9(25), p. 443-464.

7. Maisonnave H. et Decaluwe B. (2010). Politique éducative et marché $d u$ travail en Afrique du Sud. Une analyse en EGC. Recherches économiques de Louvain. 76(3), p. 289-335.

8. Diebolt C. (2000). Dépenses d'éducation et cycles économiques en Espagne aux XIXème et XXème siècles. L'Harmattan, Paris.

9. Edeh C. E., Obi A. and Obi C. O. (2018). Impact of Education Spending on Poverty Reduction in a Democratic Dispensation in 
Nigeria. International Journal of Economics and Financial Management. 3(3), p. 1-9.

10. Ferreira A. C. P. (2013). Le système éducatif du Cap Vert : réformes et enjeux actuels. Revue internationale d'éducation de Sèvres. [En ligne], 63 | septembre 2013, mis en ligne le 01 septembre 2015. URL : http://journals.openedition.org/ries/3458 ; DOI : 10.4000/ries.3458

11. FMI (2017). L'économie informelle en Afrique subsaharienne. Perspectives Economiques régionales : Afrique Subsaharienne, p. 5373.

12. Foko B., Tiyab B. K. et Husson G., (2012). Les Dépenses des ménages en éducation: une perspective analytique et comparative pour 15 pays d'Afrique. Dans rapport UNESCO Office Dakar and Regional Bureau for Education in Africa, 2012.

13. Herrera R. (1998). Dépenses publiques d'éducation et capital humain dans un modèle convexe de croissance endogène. Revue économique, 49(3), p. 831-844.

14. Honlonkou A. (2003). Corruption, inflation, croissance et développement humain durable. Y a-t-il un lien ? Mondes en développement. 123(3), p. 89-106.

15. Hysa E. (2011). Corruption and Human Development: Albania and $E U$-27. Social Studies, 5(2), p. 43-51.

16. Johansson A., Heady C., Arnold J. (2008). Taxation and Economic Growth. OECD Economics Department Working Papers, ${ }^{\circ} 620$.

17. Krueger A. B. and Lindahl M. (2000). Education for Growth: Why and for Whom? Journal of Economic Literature, 39(4), p. 01-36.

18. Kumar M. S. and Woo J. (2010). Public Debt and Growth. IMF Working Paper, July, 2010, p. 01-47.

19. Murshed M., Awlad Merdula F. A. (2018). Impacts of Corruption on Sustainable Development: A Simultaneous Equations Model Estimation Approach. Journal of Accounting, Finance and Economics. 8(1), p.109-133.

20. Reinikka R. and Svensson J., (2004). Local capture: evidence from a central government transfer program in uganda. The Quarterly Journal of Economics, 119 (2).

21. Romer P. (1990). Human capital and growth: theory and evidence. Carnegie Rochester Conference Series on Public Policy 32, p. 251286.

22. Siggel E. (2000). Uganda's policy Reforms, Competitiveness and regional integration industry: A comparison with Kenya. African Economic Policy. Discussion paper 24; Washington: United States Agency for International Development: Bureau for Africa. 
23. Siggel E. (2001). India's trade policy Reforms and Competitiveness industry in the 1980s. World Economy, p.159-183.

24. Sow A. (2013). La contribution de l'éducation à la croissance économique du Sénégal. Thèse. Université Gaston Berger.

25. Spence M. (1973). Job Market Signaling. The Quarterly Journal of Economics. 87(3), p. 355-374.

26. Toure D. (2020). Enseignement supérieur, capital humain et croissance économique: Une approche par l'analyse causale. European Scientific Journal. 16(16), p. 255-280.

27. UNESCO (2018). Tenir nos engagements en faveur de l'égalité des genres dans l'éducation. Rapport mondial de suivi sur l'éducation.

28. UNESCO (2019). Migration, déplacement et éducation : bâtir des ponts, pas des murs. Rapport mondial de suivi sur l'éducation.

29. UNESCO (2020). Inclusion et éducation : tous, sans exception. Rapport mondial de suivi de l'éducation. 